\title{
Excitonic magnetism at the intersection of spin-orbit coupling and crystal-field splitting
}

\author{
Teresa Feldmaier, ${ }^{1}$ Pascal Strobel, ${ }^{1}$ Michael Schmid, ${ }^{1,2}$ Philipp Hansmann,,${ }^{3,4}$ and Maria Daghofer ${ }^{1,2}$ \\ ${ }^{1}$ Institute for Functional Matter and Quantum Technologies, University of Stuttgart, 70550 Stuttgart, Germany \\ ${ }^{2}$ Center for Integrated Quantum Science and Technology, University of Stuttgart, 70550 Stuttgart, Germany \\ ${ }^{3}$ Max-Planck-Institute for Chemical Physics of Solids, 01187 Dresden, Germany \\ ${ }^{4}$ Department of Physics, University of Erlangen-Nuremberg, 91058 Erlangen, Germany
}

(Received 11 November 2019; revised 16 June 2020; accepted 17 June 2020; published 5 August 2020)

\begin{abstract}
Excitonic magnetism involving superpositions of singlet and triplet states is expected to arise for two holes in strongly correlated and spin-orbit-coupled $t_{2 g}$ orbitals. However, uncontested material examples for its realization are rare. We apply the variational cluster approach to the square lattice to investigate excitonic antiferromagnetism and the impact of a crystal field. We give a phase diagram depending on spin-orbit coupling and crystal field and find excitonic magnetism to survive in the presence of substantial crystal-field-induced orbital order. We address the specific example of $\mathrm{Ca}_{2} \mathrm{RuO}_{4}$ using ab initio modeling and conclude it to realize such excitonic magnetism despite its pronounced orbital polarization. We also reproduce magnetic excitations and show the maximum at momentum $(0,0)$ to be related to the excitonic character of the magnetic order.
\end{abstract}

DOI: 10.1103/PhysRevResearch.2.033201

\section{INTRODUCTION}

Strong correlations in transition-metal oxides have for decades been a focus of intense scientific activity. While the prominent $3 d$ metals, e.g., iron, nickel, and copper, are usually understood to have rather weak spin-orbit coupling (SOC), the interplay of strong SOC and correlations has attracted attention more recently. One important driver of this interest is the search for topologically nontrivial phases like topological Mott insulators [1] or spin liquids. In particular, honeycomblattice compounds with a single hole in strongly correlated $t_{2 g}$ orbitals of $4 d$ (e.g., Ru) or $5 d$ (e.g., Ir) elements are candidates for "Kitaev" spin liquids [2] driven by distinct magnetic anisotropies $[3,4]$, which has led to extensive research efforts $[5,6]$.

The impact of SOC is particularly transparent for a $t_{2 g}$ electron, where it couples effective orbital angular momentum $L=1$ and $\operatorname{spin} S=\frac{1}{2}$ to total angular momentum $J=\frac{3}{2}$ or $J=\frac{1}{2}$ [7]. If sufficiently large, then this leads to a band splitting and the reduced width of the resulting subbands effectively enhances correlations. The single-layer squarelattice compound $\mathrm{Sr}_{2} \mathrm{IrO}_{4}$ has been established as a prime example for such spin-orbit driven Mott physics [8]. With five $t_{2 g}$ electrons, its $J=\frac{3}{2}$ levels are completely filled and the $J=\frac{1}{2}$ level is half filled. The resulting effective singleband description focusing on the $J=\frac{1}{2}$ states is even robust enough to survive in doped regimes, where Fermi arcs [9] and hints of superconductivity [10] have been reported, as well as photodoping [11].

Published by the American Physical Society under the terms of the Creative Commons Attribution 4.0 International license. Further distribution of this work must maintain attribution to the author(s) and the published article's title, journal citation, and DOI.
While a description in terms of $J=\frac{1}{2}$ is thus well established for a single hole in the $t_{2 g}$ levels of $\mathrm{Ru}$ or Ir and forms the basis for the proposed spin-liquid scenario, the situation for two holes is less clear. Double-perovskite iridates $[12,13]$ likely realize a nonmagnetic singlet ground state and might already be close to the $j$ - $j$-coupling regime with a doubly occupied $J=\frac{1}{2}$ band [14]. Theoretical predictions for substantial but not extreme spin-orbit coupling, where $L-S$ coupling is more appropriate, argue that it combines effective angular momentum $L=1$ with total spin $S=1$ of the two holes into an ionic ground state with $J=0[15,16]$, see the left-hand side of Fig. 1(a). Superexchange then determines dynamics of $J=1$ excitations as well as their potential condensation into excitonic magnetic order. This unconventional type of magnetism has been predicted to host a bosonic Kitaev-Heisenberg model and topologically nontrivial excitations $[17,18]$ or to mediate triplet superconductivity [19].

$\mathrm{Ca}_{2} \mathrm{RuO}_{4}$ is a promising candidate for such a scenario, where superexchange mixes $J=0$ and $J=1$ states so that the superposition acquires a magnetic moment that can order; see the schematic illustration in Fig. 1(c). Features like $X Y$ symmetry breaking (rather than Ising or Heisenberg) and the corresponding maximum of excitation energies at momentum $(0,0)$ can be explained by such an excitonic model [20,21] However, a structural phase transition is well established $[22,23]$, which leads to a crystal field (CF) lowering the energy of the $x y$ orbital as shown on the right-hand side of Fig. 1(a). Based on $a b$ initio calculations [24,25], the two holes are distributed mostly in $x z$ and $y z$ orbitals, suggesting more conventional $S=1$ spin order; see the sketch of Fig. 1(d). $\mathrm{SOC}$ would then be a correction rather than a main driver [24,26].

We apply here the variational cluster approach (VCA) to a spin-orbit coupled and correlated $t_{2 g}$ three-orbital model at nominal filling $n=4$ in order to address the competition between SOC and CF as illustrated in Fig. 1. We find that 


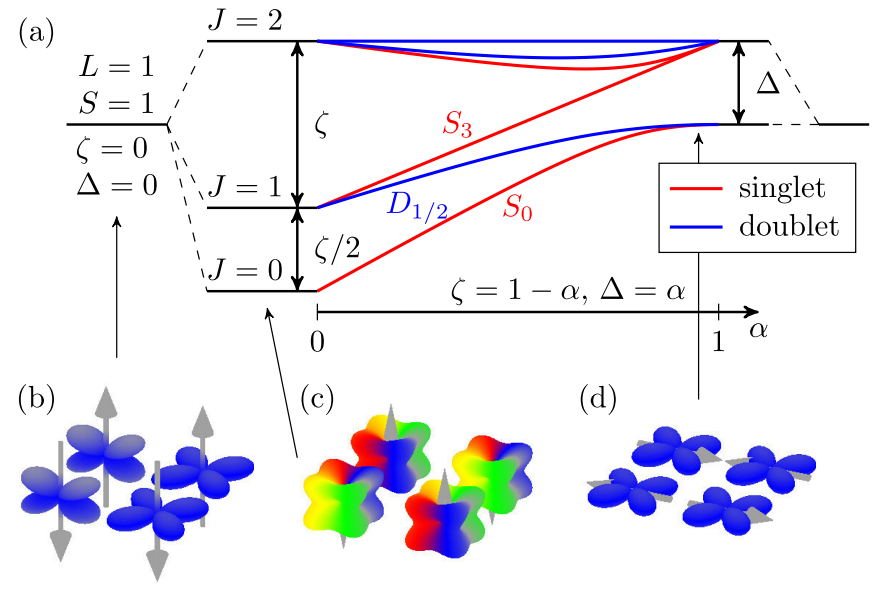

FIG. 1. (a) Level structure starting from ninefold degeneracy (far left and right) and interpolating between spin-orbit-coupled (left, $\alpha=0$ ) and crystal field (CF) split (right, $\alpha=1$ ) regimes. In the limit $\Delta=\alpha=0$ (left), singlet $S_{3}$ and doublet $D_{1 / 2}$ are degenerate and give the $J=1$ triplet; in the limit $\zeta=1-\alpha=0$ (right), groundstate singlet $S_{0}$ and $D_{1 / 2}$ correspond to $S=1$ and doubly filled $x y$ orbital. (b) The stripy state arising out of ninefold degeneracy via Goodenough-Kanamori rules. (c) Excitonic magnetism for dominant SOC, one state of the $J=1$ triplet condenses to yield a magnetic moment and mixes with the $J=0$ singlet. (d) Orbitally ordered state with doubly occupied $x y$ orbital, where holes in $x z$ and $y z$ form $S=1$. In (b) and (d), the doubly occupied orbital is drawn.

without $\mathrm{CF}$ splitting, SOC $\zeta$ suppresses orbital order, so that the complex spin and orbital pattern of Fig. 1(b) is replaced by the excitonic antiferromagnetic (AFM) state Fig. 1(c). In the presence of CF splitting, its impact will be seen to be more gradual, but it nevertheless induces a transition from spin order, as in Fig. 1(d), to excitonic order. In order to connect to $\mathrm{Ca}_{2} \mathrm{RuO}_{4}$, we use a realistic model based on density-functional theory and subsequent projection to Wannier states [27]. The approach is validated by the magnetic excitation spectrum of a spin-orbital model based on the same parameters, which reproduces neutron-scattering data, and leads to the conclusion that the compound falls into the excitonic regime despite its strong orbital polarization.

In Sec. II, we introduce the $t_{2 g}$ model with SOC and also give the Kugel-Khomskii-like superexchange model valid in the limit of strong onsite interactions in Sec. II B. We also shortly mention the VCA. We then give results in Sec. III, first for the orbitally degenerate model in Sec. III A and then in the presence of a crystal field in Sec. III B, where we also discuss $\mathrm{Ca}_{2} \mathrm{RuO}_{4}$. Finally, we provide magnetic excitation spectra in Sec. III C and conclude in Sec. IV.

\section{MODEL AND METHODS}

We discuss four electrons (two holes) in a three-orbital system modeling $t_{2 g}$ orbitals on a square lattice. The kinetic energy

$$
H_{\mathrm{kin}}=\sum_{\mathbf{k}, \sigma} \sum_{\alpha, \beta} \epsilon_{\alpha, \beta}(\mathbf{k}) c_{\mathbf{k}, \alpha, \sigma}^{\dagger} c_{\mathbf{k}, \beta, \sigma},
$$

where $c_{\mathbf{k}, \alpha, \sigma}\left(c_{\mathbf{k}, \alpha, \sigma}^{\dagger}\right)$ annihilates (creates) an electron with spin $\sigma=\uparrow, \downarrow$ in orbital $\alpha=x y, x z, y z$ and momentum $\mathbf{k}$, has the following contributions:

$$
\begin{aligned}
\epsilon_{x y, x y}(\mathbf{k})= & -2 t_{x y}^{\mathrm{NN}}\left(\cos k_{x}+\cos k_{y}\right) \\
& -4 t_{x y}^{\mathrm{NNN}} \cos k_{x} \cos k_{y}-\Delta,
\end{aligned}
$$

where tetragonal CF splitting $\Delta>0$ originates from the strained octahedral coordination of the low-temperature phase of $\mathrm{Ca}_{2} \mathrm{RuO}_{4}$,

$$
\epsilon_{x z, x z}(\mathbf{k})=-2 t_{x z}^{\mathrm{NN}} \cos k_{x}, \quad \epsilon_{y z, y z}(\mathbf{k})=-2 t_{y z}^{\mathrm{NN}} \cos k_{y},
$$

and

$$
\epsilon_{x z, x y}(\mathbf{k})=-2 t^{o} \cos k_{x},
$$

where orbital-mixing $t^{o}$ arises from the orthorhombic distortion. The SOC operator for $t_{2 g}$ orbitals can be written as [28,29]

$$
H_{\mathrm{SOC}}=\zeta \sum_{i} \mathbf{l}_{i} \cdot \mathbf{s}_{i}=\frac{i \zeta}{2} \sum_{i} \sum_{\substack{\alpha, \beta, \gamma \\ \sigma, \sigma^{\prime}}} \varepsilon_{\alpha \beta \gamma} \tau_{\sigma \sigma^{\prime}}^{\alpha} c_{i, \beta, \sigma}^{\dagger} c_{i, \gamma, \sigma^{\prime}},
$$

where $\varepsilon_{\alpha \beta \gamma}$ is the totally antisymmetric Levi-Civita tensor and $\tau^{\alpha}$ with $\alpha=x, y, z$ are Pauli matrices.

The effective onsite two-particle interaction is included in the SU(2) symmetric Kanamori approximation [30],

$$
\begin{aligned}
H_{\mathrm{int}}= & U \sum_{i, \alpha} n_{i \alpha \uparrow} n_{i \alpha \downarrow}+\frac{U^{\prime}}{2} \sum_{i, \sigma} \sum_{\alpha \neq \beta} n_{i \alpha \sigma} n_{i \beta \bar{\sigma}} \\
& +\frac{1}{2}\left(U^{\prime}-J_{H}\right) \sum_{i, \sigma} \sum_{\alpha \neq \beta} n_{i \alpha \sigma} n_{i \beta \sigma} \\
& -J_{H} \sum_{i, \alpha \neq \beta}\left(c_{i \alpha \uparrow}^{\dagger} c_{i \alpha \downarrow} c_{i, \beta \downarrow}^{\dagger} c_{i \beta \uparrow}-c_{i \alpha \uparrow}^{\dagger} c_{i \alpha \downarrow}^{\dagger} c_{i \beta \downarrow} c_{i \beta \uparrow}\right)
\end{aligned}
$$

with intraorbital Hubbard $U$, interorbital $U^{\prime}$, and Hund's coupling $J_{H}$, which are related by $U^{\prime}=U-2 J_{H}$.

\section{A. Variational cluster approximation}

In order to address long-range order and symmetry breaking, we use the VCA [31], which has already been established to explain angle-resolved photoemission for strongly spinorbit-coupled $\mathrm{Sr}_{2} \mathrm{IrO}_{4}$ [32]. VCA is complementary to static mean-field methods, which cannot describe the large- $U$ limit of $t_{2 g}$ orbitals with SOC correctly [33], and dynamical meanfield theory with Monte Carlo impurity solvers which are restricted to regimes above the Néel temperature due to the minus-sign problem [27]. The VCA is further not restricted to the one-dimensional limit addressed with the density-matrix renormalization group [33,34].

In our VCA simulations, we use (Lanczos) exact diagonalization (ED) to obtain the cluster Greens function $G_{\mathrm{Cl}}$ of a $2 \times 2$-sites cluster, extract the cluster self-energy $\Sigma$, and insert it into the one-particle Green's function $G$ of the thermodynamic limit. The approximation thus consists of replacing the self-energy in $G$ by the self-energy of the cluster. The grand potential is calculated from this approximated Green's function and from the grand potential $\Omega_{\mathrm{Cl}}$ of the cluster as

$$
\Omega=\Omega_{\mathrm{Cl}}+\operatorname{tr} \ln G-\operatorname{tr} \ln G_{\mathrm{Cl}} .
$$


The chemical potential $\mu$

$$
-\mu N:=-\mu \sum_{i, \alpha, \sigma} n_{i, \alpha, \sigma},
$$

where the sum runs over over site $i$, orbital $\alpha$, and spin $\sigma$ should here stabilize a filling of $N_{\text {target }}=2$ holes, i.e., four electrons, per site. The optimal $\mu$ can be determined by going from $\Omega$ to the free energy $F=\Omega+\mu N_{\text {target }}$ via a Legendre transform [35], so that stationarity

$$
\frac{\partial F}{\partial \mu}=\frac{\partial \Omega+\mu N_{\text {target }}}{\partial \mu}=0
$$

indeed translates to $N_{\text {target }}=\langle N\rangle$. The chemical potential $\mu$ then becomes a variational parameter.

According to self-energy functional theory [36], the thermodynamic grand potential $\Omega\left[\Sigma_{\tau^{\prime}}\right]$ can moreover be optimized by varying any one-particle parameters $\tau^{\prime}$ used to obtain the cluster self-energy $\Sigma_{\tau^{\prime}}$. These fictitious parameters are used when the cluster Green's function $G_{\mathrm{Cl}}$ and the self-energy $\Sigma_{\tau^{\prime}}$ are calculated but are set back to their nominal values when obtaining the full Green's function $G$. For instance, the actual overall density $\langle N\rangle_{\mu^{*}}$ obtained for the saddle-point chemical potential $\mu^{*}$, which is after all approximated, does not necessarily fulfill $\langle N\rangle_{\mu^{*}}=N_{\text {target }}$. To ensure thermodynamic consistency [37], we thus additionally optimized a fictitious chemical potential $\mu^{\prime}$ that was included on the cluster but subtracted again when constructing $G$.

The self-energy is finally optimized in the same way with respect to fictitious symmetry-breaking fields, e.g., a staggered magnetic field inducing AFM order. They can generally be expressed as one-particle terms of the form

$$
H_{h}=h^{\prime} \sum_{i} \Lambda_{i} e^{i \mathbf{Q} \mathbf{R}_{i}}
$$

where $\Lambda_{i}$ can be any one-particle operator acting on site $i$ at position $\mathbf{R}_{i}$, e.g., spin $S_{i}^{\alpha}$ along direction $\alpha$, total angular momentum $S_{i}^{\alpha}+L_{i}^{\alpha}$, magnetization $2 S_{i}^{\alpha}-L_{i}^{\alpha}$ or any other linear combination of $S$ and $L$, as well as orbital density $n_{i, \beta}$ in orbital $\beta$. As with $\mu^{\prime}$, these fields only act on the reference system, they are not included in the thermodynamiclimit Green's functions. If, however, a symmetry-broken selfenergy optimizes the grand potential of the fully symmetric Hamiltonian, one can infer spontaneous symmetry breaking.

We sampled a variety of potential ordering parameters, where ordering vectors $\mathbf{Q}$ accessible to our cluster are $(0,0)$, $(\pi, \pi),(\pi, 0)$, and $(0, \pi)$. We also included superpositions of two or three order parameters, e.g., orbital and magnetic order, with identical or different (e.g., in the stripy phase) ordering vectors, see Table I. The patterns tested were motivated on one hand by the states giving a large contribution to the ED ground state and on the other hand by phases reported in the literature for similar models [38,39]. The robust Mott gap present in all considered parameter regimes ensures a very weak dependence of $\mu$ and $\mu^{\prime}$ on the various $h^{\prime}$, so that we fixed $\mu$ and $\mu^{\prime}$ to the values found for $h^{\prime}=0$ and then kept them constant during optimization of the $h^{\prime}$ [40].

One-particle observables like orbital- or spin-resolved densities can be obtained from the thermodynamic-limit oneparticle Green's function that is calculated in the VCA. Two-particle quantities are not as straightforward, and we
TABLE I. Fictitious Weiss fields $\Lambda$ and ordering momenta $\mathbf{Q}$ as

\begin{tabular}{|c|c|c|c|}
\hline & $\Lambda_{1}$ & $\mathbf{Q}_{1}$ & $\Lambda_{2}$ \\
\hline Stripy $S_{z}$ & $S_{z}$ & $(\pi, 0)$ & \\
\hline Stripy $S_{z}$, stripy orb. & \multicolumn{2}{|c|}{$\Lambda_{3}=n_{y z}$} & $\mathbf{Q}_{3}=(0,0)$ \\
\hline Stripy $S_{x}$ & $S_{x}$ & $(\pi, 0)$ & \\
\hline Stripy $S_{x}$, stripy orb. & \multicolumn{2}{|c|}{$\Lambda_{3}=n_{y z}$} & $\begin{array}{c}n_{x y}-n_{x z} \quad(0, \pi) \\
\mathbf{Q}_{3}=(0,0)\end{array}$ \\
\hline$G$-AFM $S_{z}$ & $S_{z}$ & $(\pi, \pi)$ & \\
\hline$G$-AFM $S_{x}$ & $S_{x}$ & $(\pi, \pi)$ & \\
\hline$G$-AFM $S_{z}$, FO $x y$ & $S_{z}$ & $(\pi, \pi)$ & $n_{x y} \quad(0,0)$ \\
\hline$G$-AFM $S_{z}$, TO & $S_{z}$ & $(\pi, \pi)$ & orb. see Ref. [38] \\
\hline$G$-AFM $2 S_{z}+L_{z}$ & $2 S_{z}+L_{z}$ & $(\pi, \pi)$ & \\
\hline$G$-AFM $2 S_{x}+L_{x}$ & $2 S_{x}+L_{x}$ & $(\pi, \pi)$ & \\
\hline$G$-AFM $M_{z}$ & $2 S_{z}-L_{z}$ & $(\pi, \pi)$ & \\
\hline$G$-AFM $M_{x}$ & $2 S_{x}-L_{x}$ & $(\pi, \pi)$ & \\
\hline$G$-AFM $J_{z}$ & $S_{z}+L_{z}$ & $(\pi, \pi)$ & \\
\hline$G-\operatorname{AFM} J_{x}$ & $S_{x}+L_{x}$ & $(\pi, \pi)$ & \\
\hline$G$-AFM $S_{x}+S_{y}$ & $S_{x}+S_{y}$ & $(\pi, \pi)$ & \\
\hline
\end{tabular}
in Eq. (10), as well as combinations thereof used to describe various phases.

approximate them here as the expectation value with respect to the ED ground state $\left|\phi_{0}\right\rangle$ calculated with optimized order parameters. In particular, we are interested in the weight found in eigenstates $\left|J, J^{z}\right\rangle$ with total onsite angular momentum $J=0,1,2$, i.e., in

$$
\langle J\rangle:=\frac{1}{N_{\text {sites }}} \sum_{i, J^{z}}\left\langle\phi_{0} \mid J_{i}, J_{i}^{z}\right\rangle\left\langle J_{i}, J_{i}^{z} \mid \phi_{0}\right\rangle,
$$

where we average over the $N_{\text {sites }}$ sites $i$ of the $2 \times 2$ cluster. Analogously, one can define weight in other onsite two-hole states, e.g., the states $S_{0}, D_{1 / 2}, S_{3}, \ldots$, of the level structure shown in Fig. 1(a).

\section{B. Perturbation theory and Kugel-Khomskii-type model}

While the VCA provides one-particle spectral functions, two-particle quantities like the dynamic magnetic structure factor are not readily available. We thus resort to ED on a small cluster. In order to go beyond the $2 \times 2$ cluster, we restrict the Hilbert space to the nine low-energy states of Fig. 1. In this low-energy manifold, the two holes on each site reside in two different orbitals, i.e., we conveniently label the orbital wave function by the remaining doubly occupied orbital. This gives us the effective orbital angular momentum $L=1[15,41]$. Additionally, Hund's-rule coupling enforces a total spin $S=1$, leading to a total of nine low-energy states. The VCA results show that these nine states indeed capture nearly all the weight of the cluster ground state, which justifies projecting out charge excitations as well as states with onsite spin $S=0$. The restricted Hilbert space then allows us to reach eight rather than four sites in exact diagonalization.

The effective Hamiltonian acting on this low-energy manifold is obtained by treating hopping $t$ in second-order perturbation theory, with the large energy scale being onsite interactions (6), associated with charge excitations and local singlets. We give here the various terms of the effective 
Kugel-Khomskii-type Hamiltonian, restricting ourselves to orbital-conserving hoppings for simplicity, i.e., we neglect interorbital $t^{o}$ which would significantly complicate the superexchange model. The first possible process is a spin-spin coupling

$$
H_{\mathbf{S} \cdot \mathbf{S}}=J_{i, j}\left(\mathbf{S}_{i} \mathbf{S}_{j}-1\right) \otimes\left|T_{i} ; T_{j}\right\rangle\left\langle T_{i} ; T_{j}\right|
$$

that leaves orbital occupations $T_{i}$ and $T_{j}$ unchanged. Its coupling strength depends on whether the same orbital $\gamma$ is doubly occupied on both sites, or whether it is orbital $\alpha$ on one site and orbital $\beta$ on the other, i.e.,

$$
J_{i, j}= \begin{cases}\left(t_{\alpha}^{2}+t_{\beta}^{2}\right) \frac{U+J_{H}}{U\left(U+2 J_{H}\right)} & \text { for } T_{i}=T_{j}=\gamma \\ \frac{t_{\gamma}^{2}\left(U+J_{H}\right)}{U\left(U+2 J_{H}\right)}-\frac{J_{H}\left(t_{\alpha}^{2}+t_{\beta}^{2}\right)}{U\left(U-3 J_{H}\right)} & \text { for } T_{i}=\alpha \neq T_{j} \\ & =\beta \text { and } \alpha, \beta \neq \gamma\end{cases}
$$

and accordingly

$$
\begin{aligned}
& H_{\mathbf{S} . \mathbf{S}}=\left(\mathbf{S}_{i} \mathbf{S}_{j}-1\right)\left[\sum_{\gamma}\left(\sum_{\alpha \neq \gamma} t_{\alpha}^{2}\right) \frac{U+J_{H}}{U\left(U+2 J_{H}\right)}|\gamma ; \gamma\rangle\langle\gamma ; \gamma|\right. \\
& \left.+\sum_{\alpha \neq \beta}\left(\frac{t_{\gamma}^{2}\left(U+J_{H}\right)}{U\left(U+2 J_{H}\right)}-\frac{J_{H}\left(t_{\alpha}^{2}+t_{\beta}^{2}\right)}{U\left(U-3 J_{H}\right)}\right)|\alpha ; \beta\rangle\langle\alpha ; \beta|\right] .
\end{aligned}
$$

The first of these would be the only superexchange term surviving in a strongly orbitally polarized case for crystal field $\Delta \gg \zeta$.

Additional terms involve the orbital degree of freedom. Again discussing first the case of identical orbital character on both sites, finite Hund's-rule coupling $J_{H}>0$ (or rather the associated pair-hopping term) allows the doubly occupied orbital to change on both sites simultaneously, as long as not all spins are parallel, yielding a "pair-flip" term

$$
H_{p}=\left(\mathbf{S}_{i} \mathbf{S}_{j}-1\right)\left[\sum_{\alpha \neq \beta} \frac{-t_{\alpha} t_{\beta} J_{H}}{U\left(U+2 J_{H}\right)}|\alpha ; \alpha\rangle\langle\beta ; \beta|\right] .
$$

The counterpart for different orbital occupation on both sites gives a diagonal part, in addition to the diagonal terms already included in $S_{i}^{z} S_{j}^{z}$ above, that is independent of the $S^{z}$ components on the two sites and reads

$$
\begin{aligned}
\left\langle S_{i}^{z}, T_{i}\right. & \left.=\beta ; S_{j}^{z}, T_{j}=\gamma|H| S_{i}^{z}, T_{i}=\beta ; S_{j}^{z}, T_{j}=\gamma\right\rangle \\
& =-\frac{t_{\beta}^{2}+t_{\gamma}^{2}}{U-3 J_{H}} .
\end{aligned}
$$

This diagonal term can be combined with orbital and spinorbital-flip terms into

$$
\begin{aligned}
H_{\mathbf{T T}}= & -\sum_{\beta \neq \gamma} \frac{t_{\beta}^{2}+t_{\gamma}^{2}}{U-3 J_{H}}|\beta ; \gamma\rangle\langle\beta ; \gamma| \\
& +\sum_{\beta \neq \gamma} \frac{t_{\beta} t_{\gamma}}{U\left(U-3 J_{H}\right)}\left[2 J_{H}+\left(U-J_{H}\right)\left(\mathbf{S}_{i} \mathbf{S}_{j}+1\right)\right]
\end{aligned}
$$

$$
\otimes|\beta ; \gamma\rangle\langle\gamma ; \beta| \text {. }
$$

The full superexchange Hamiltonian for a pair $(i, j)$ of sites combines (spin-)orbital exchange (18) and (19) with Heisenberg spin interaction Eqs. (14) and (15) as well as (16) into

$$
H_{i, j}=H_{\mathbf{S} \cdot \mathbf{S}}+H_{p}+H_{\mathbf{T T}} .
$$

For each nearest-neighbor (NN) pair $\langle i, j\rangle$, two of the three hoppings $t_{\alpha / \beta / \gamma}$ are nonzero; for next-nearest neighbors $\langle\langle i, j\rangle\rangle$, only the $x y$ orbital hops.

These intersite coupling terms due to hopping are complemented with the onsite SOC and CF. Crystal field $\Delta$ from Eq. (2) favors the $x y$ orbital to be doubly occupied, which is chosen as the $L^{z}=0$ state, and SOC Eq. (5) favors the $J=0$ state, so that the onsite Hamiltonian becomes

$$
H_{\text {ion }}=\frac{\zeta}{2} \mathbf{S L}+\Delta\left(L^{z}\right)^{2}
$$

after projection onto the low-energy $S=1$ manifold. Eigenstates of this onsite Hamiltonian define the level structure shown in Fig. 1.

The translation between the notation $|\beta\rangle$ giving the doubly occupied orbital $\beta=y z, x z, x y$, as used in the superexchange model above, and the orbital angular-momentum operators is achieved by $[15,41]$

$$
\begin{aligned}
& L^{x}=i(|x y\rangle\langle x z|-| x z\rangle\langle x y|), \\
& L^{y}=i(|y z\rangle\langle x y|-| x y\rangle\langle y z|), \\
& L^{z}=i(|x z\rangle\langle y z|-| y z\rangle\langle x z|) .
\end{aligned}
$$

The operator $\left(L^{z}\right)^{2}=|x z\rangle\langle x z|+| y z\rangle\langle y z|$ thus indeed favors doubly occupied $|x y\rangle$ states.

We use Lanczos exact diagonalization to obtain spectra

$$
M^{\alpha}(\mathbf{k}, \omega)=-\frac{1}{\pi} \Im\left\langle\phi_{0}\left|M^{\alpha}(-\mathbf{k}) \frac{1}{\omega-H+\mathrm{i} 0^{+}} M^{\alpha}(\mathbf{k})\right| \phi_{0}\right\rangle,
$$

where $M^{\alpha}(\mathbf{k})=\frac{1}{N} \sum_{i} e^{i \mathbf{k r}_{i}} M_{i}^{\alpha}$ for component $\alpha=a, b, c$ of $\mathbf{M}=2 \mathbf{S}-\mathbf{L}$ and effective low-energy $H$ composed of onsite and intersite terms (21) and (20). We are able to treat an eightsite cluster of $\sqrt{8} \times \sqrt{8}$ geometry. 


\section{RESULTS}

We are first going to discuss a more generic model for excitonic magnetism with and without CF splitting, where we focus on essentials. We choose $t=t_{x y}^{\mathrm{NN}}=t_{x z}^{\mathrm{NN}}=t_{y z}^{\mathrm{NN}}$ as unit of energy and set $t_{x y}^{\mathrm{NNN}}=t^{o}=0$. Interactions are fixed to $U=12.5 t$ and $J_{H}=2.5 t$, which is consistent with their order of magnitude in $\mathrm{Ca}_{2} \mathrm{RuO}_{4}$ [25] and solidly in the Mott insulating regime. $\mathrm{SOC} \zeta$ and $\mathrm{CF} \Delta$ will be varied in order to assess their impact.

\section{A. Excitonic AFM ordering without CF}

Figure 2 shows how much the VCA grand potential $\Omega$ is lowered by symmetry breaking with respect to the paramagnetic (PM) phase for various potential order parameters. Crystal field is here $\Delta=0$ and the ground-state ordering for each $\zeta$ is the one giving the lowest $\Omega$. For $\zeta>0$, the out-ofplane $z$ component and the in-plane $x$ component of angular momentum lead to clearly different values, reflecting the loss of rotational symmetry. Different linear combinations of $S$ and $L$, on the other hand, typically only give slightly different grand potentials - the crucial aspect of the order parameter is the symmetry broken rather than the exact form of the order parameter.

For vanishing or small SOC, the lowest grand potential is obtained for a complex pattern with orbital and spin order having orthogonal ordering vectors $(0, \pi)$ and $(\pi, 0)$, as depicted in Fig. 1(b). Such a state is consistent with Goodenough-

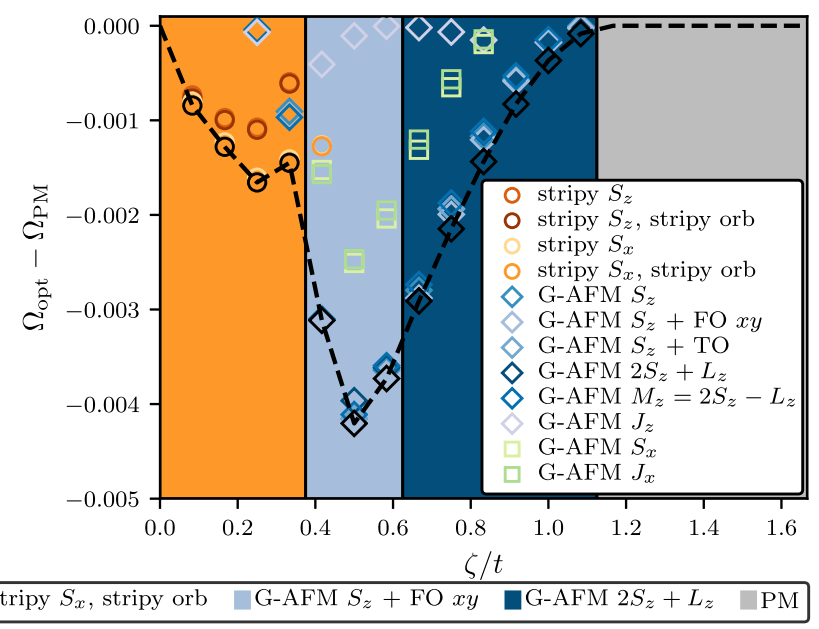

FIG. 2. Grand-canonical potential per site obtained with VCA for various order parameters in units of $t . \Omega_{\mathrm{PM}}$ is the value obtained without symmetry breaking and $\Omega_{\mathrm{opt}}$ is the lowest grand potential found by allowing for various ordered states, see Table I: " $G$-AFM" refers to checkerboard pattern, i.e., $\mathbf{Q}=(\pi, \pi)$ in Eq. (10), "stripy" to $\mathbf{Q}=(0, \pi)$, and "FO" to ferrorobital order $[\mathbf{Q}=(0,0)]$ favoring the $x y$ orbital, "TO" is the complex three-orbital-order of Ref. [38]. "Stripy orb." denotes the pattern of Fig. 1(b), with $\mathbf{Q}_{\text {orb }}=(\pi, 0)$ orthogonal to $\mathbf{Q}_{\text {spin }}=(0, \pi)$ of the concurrent stripy spin pattern. The dashed line is a guide to the eye connecting the lowest- $\Omega$ values, whose color is used for the background. A gray background denotes the paramagnetic regime, where no symmetry breaking lowers the grand potential. Parameters are $t_{x y}^{\mathrm{NN}}=t_{x z}^{\mathrm{NN}}=t_{y z}^{\mathrm{NN}}=t, \Delta=0, U=$ $12.5 t$, and $J_{H}=2.5 t$.

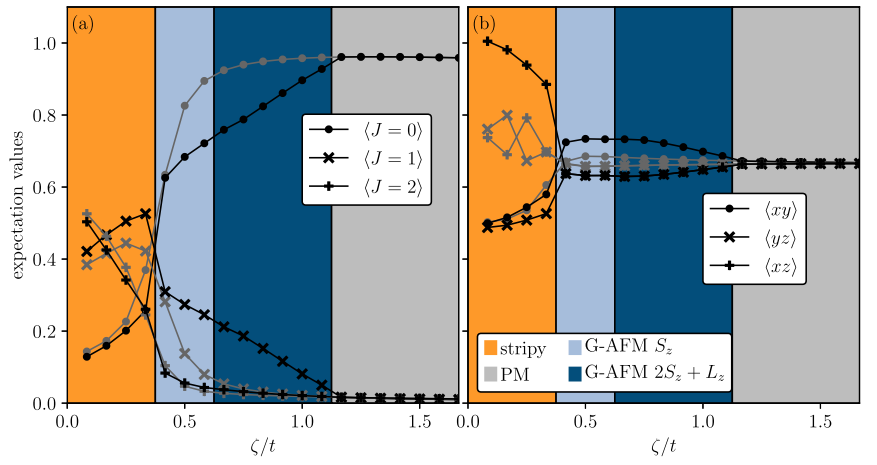

FIG. 3. Spin-orbital onsite state and orbital occupations for parameters as in Fig. 2, i.e., without crystal field. Gray symbols give (a) weights Eq. (11) found in states with total onsite angular momentum $J=0,1,2$ and (b) orbital occupation numbers $n_{y z / x z / x y}^{h}$ for holes. Black symbols indicate the same observables but obtained for the ordered state, i.e., with optimized symmetry-breaking cluster parameters as inferred from Fig. 2. Background color labels the ordered phase yielding the lowest grand potential as in Fig. 2: orange (stripy) [see Fig. 1(b) but with in-plane spins], blue (checkerboard) $G$-AFM order with out-of-plane moment (light blue: $S_{z}$ plus a small $x y$ polarization; dark blue: $2 S_{z}+L_{z}$ ), and gray (paramagnet).

Kanamori rules, has also been found by ED [39], and remains stable for $\zeta \lesssim 0.4 t$. The only role of SOC is here a slight preference for $S^{x}$ over $S^{z}$. At $\zeta \approx 0.4 t$, the magnetic ordering vector switches to $(\pi, \pi)$ in a first-order transition, see Fig. 2, and orbital densities become more equal as stripy orbital order is lost, see Fig. 3(b).

With the transition to checkerboard order, total onsite angular momentum $J$ becomes more relevant, as can be inferred from the weight (11) in eigenstates $|J=0,1,2\rangle$. The $J=0$ state quickly gains weight with the onset of checkerboard AFM order, as shown in Fig. 3(a) and similarly seen using dynamical mean-field theory [42]. In fact, it almost entirely describes the PM state already for $\zeta \approx 0.6 t$, see the gray symbols in Fig. 3(a). Magnetic ordering increases weight in $J=1$ (see black symbols), exactly as expected for excitonic magnetism, while the $J=2$ are nearly irrelevant in the entire checkerboard regime. The small orbital polarization $n_{x y}^{h}>$ $n_{x z}^{h}, n_{y z}^{h}$, see Fig. 3(b) is due to the layered geometry and favors an ordered moment along $z$ rather than in plane. The onset of checkerboard order together with the shift to the $J=0$ state suggests that ordering vector $(\pi, \pi)$ is favored as soon as $\zeta$ sufficiently lifts orbital degeneracy.

\section{B. Competition of SOC and CF}

Having established the signatures of excitonic magnetism in the case of degenerate orbitals, we now include a tetragonal CF splitting that favors doubly occupied $x y$ orbitals and thus breaks orbital degeneracy from the outset. This is the situation depicted in Fig. 1(d), where the two holes occupy $x z$ and $y z$ orbitals and form a total spin one. As can be inferred from the VCA grand potential shown in Fig. 4, checkerboard AFM order arises as expected for $\zeta=0$. Orbital-resolved hole densities in Fig. 5(a) show that the CF splitting $\Delta=1.5 t$ is 


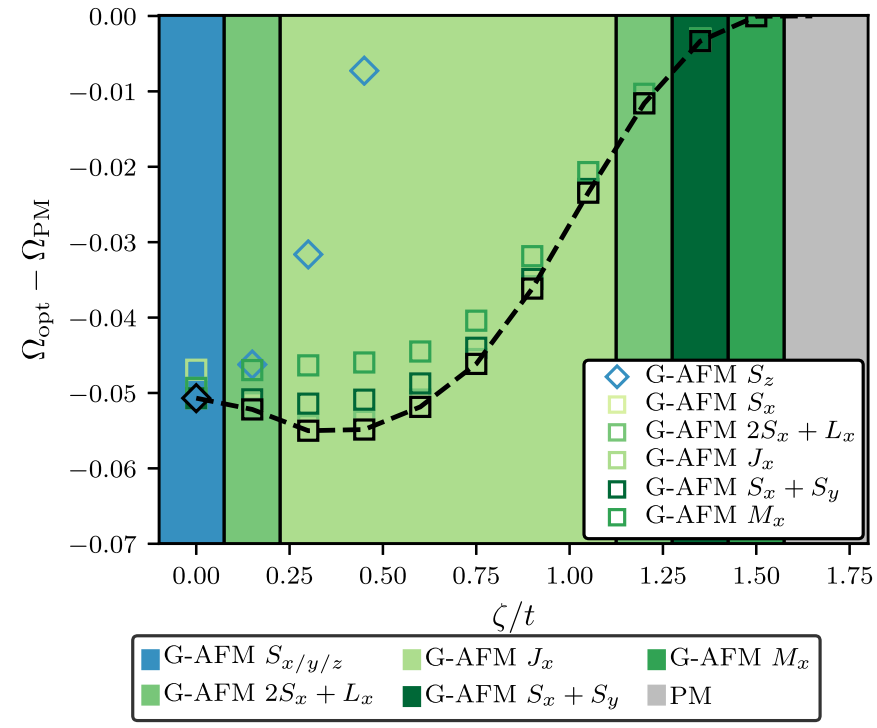

FIG. 4. Grand-canonical potentials obtained with VCA with crystal field $\Delta=1.5 t$, remaining parameters as in Fig. 2. All magnetic ordering vectors are $\mathbf{Q}_{\text {magn }}=(\pi, \pi)$ and the substantial CF suppresses any orbital order, so that corresponding Weiss fields are all 0 .

strong enough to completely fill the $x y$ orbital, so that we find pure spin-AFM without any excitonic character.

Once SOC $\zeta$ sets it, the in-plane $x$ component of the spin rapidly becomes favorable over the $z$ component, see the grand potentials in Fig. 4. At the same time, hole density $n_{x y}^{h}$ in $x y$ increases with SOC, see Fig. 5(a), but remains rather low with $n_{x y}^{h} \lesssim 0.2$ vs $n_{x z / y z}^{h} \gtrsim 0.9$ for $\zeta \lesssim t$. However, for even stronger $\zeta>1.5 t$, magnetic symmetry breaking does not improve the grand potential, see Fig. 4. This complete suppression of AFM order clearly indicates a substantial role of SOC, even though orbital polarization continues to be substantial with $n_{x y}^{h} \approx 0.4$ vs $n_{x z / y z}^{h} \approx 0.8$.

In the intermediate regime between the isotropic spin$\mathrm{AFM}$ at $\zeta=0$ and the nonmagnetic state at $\zeta>1.5 t$, various linear combinations of $S$ and $L$ can have quite similar grand potentials as long as the ordered moment lies in the $x-y$ plane, see Fig. 4. While $J_{x}$ dominates over a wide range of $\zeta$, the small differences in $\Omega$ suggest that the exact form of the order parameters is again far less important than its in-plane character.

More information about the intermediate magnetic state can be gained from the observables plotted in Fig. 5. While weight (11) is moved from $J=1$ and $J=2$ states toward $J=$ 0 with growing $\zeta$, Fig. 5(b) shows that the $J=2$ states remain populated throughout, even in the PM regime at strong $\zeta>$ $1.5 t$. Nevertheless, total angular momentum shows signatures of excitonic magnetism for $\zeta \gtrsim 0.6 t$ : When comparing the gray symbols obtained in the PM state to the black ones of the AFM state, one notices a weight transfer from $J=0$ to $J=1$, while weight in $J=2$ is hardly affected. The $J=2$ states thus carry finite weight, and contribute to orbital polarization, but are not involved in the magnetic ordering.

A clearer picture emerges when weight in eigenstates of $J$ is replaced by weight in eigenstates of the effective onsite Hamiltonian (21), whose level structure is shown in Fig. 1(a).
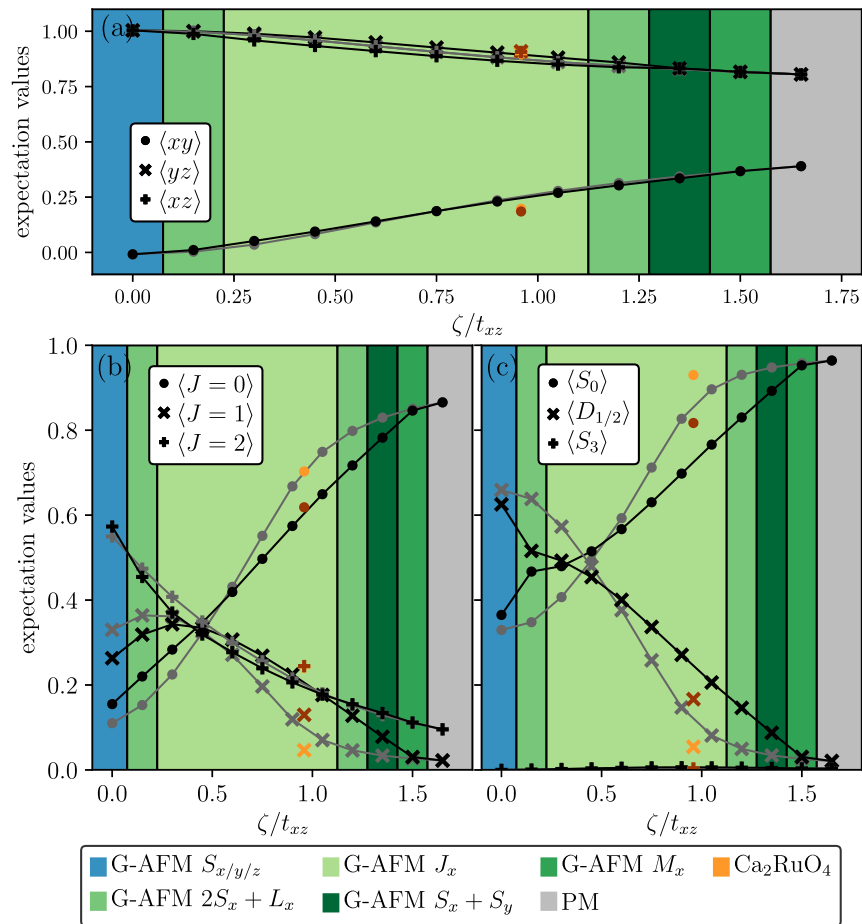

FIG. 5. Orbital occupation and spin-orbital order in the presence of a CF. (a) gives orbitally resolved hole occupation numbers $n_{y z / x z / x y}^{h}$, (b) weights (11) found in states with total onsite angular momentum $J=0,1,2$, and (c) shows analogous weights in eigenstates of (21), i.e., in the levels $S_{0}, D_{1 / 2}$, and $S_{3}$ of Fig. 1(a). Gray (black) symbols are results obtained without (with) AFM order. Background color refers to the optimal order parameter inferred from the VCA grand potentials of Fig. 4 and given below the figure; it always lies in the $x-y$ plane, except for the isotropic limit $\zeta=0$. Parameters as in Fig. 2 except that $\Delta=1.5 t$. Orange (red) symbols refer to the parameters used to model $\mathrm{Ca}_{2} \mathrm{RuO}_{4}$, see text, and the state without (with) $G$-AFM symmetry breaking, the latter prefers moments along $b=\frac{1}{\sqrt{2}}(x+y)$ direction.

Figure 5(c) shows that in the whole parameter regime, only the three lowest states $S_{0}$ and $D_{1 / 2}$ are relevant and fully capture a continuous transition from a spin-one via an excitonic magnet to the PM phase. For moderate and strong $\zeta \gtrsim 0.7 t$, magnetic ordering can be described in perfect analogy to Fig. 3(a), i.e., using the excitonic picture of weight transfer from the singlet ground state $S_{0}$ to the doublet $D_{1 / 2}$.

We now focus on the specific material example of $\mathrm{Ca}_{2} \mathrm{RuO}_{4}$ by applying the VCA to realistic hopping parameters obtained from density-functional theory and projection onto Wannier states. We use here the parameters derived in Ref. [27], see its supplemental material for details, but neglect very small hoppings. This yields $t_{x y}^{\mathrm{NN}}=0.2 \mathrm{eV}, t_{x z}^{\mathrm{NN}}=$ $t_{y z}^{\mathrm{NN}}=0.137 \mathrm{eV}, t_{x y}^{\mathrm{NNN}}=0.1 \mathrm{eV}$, and $t^{o} \approx 0.09 \mathrm{eV}$, as well as $\mathrm{CF} \Delta \approx 0.25 \mathrm{eV}$. Recent $\mathrm{x}$-ray scattering experiments have concluded that $U \approx 2 \mathrm{eV}$ and $J_{H} \approx 0.34 \mathrm{eV}$ and that oxygen covalency reduces SOC from its free-ion value $\zeta \approx 0.16 \mathrm{eV}$ to $\zeta \approx 0.13 \mathrm{eV}$ [14]. As mentioned in the supplement of Ref. [27], double-counting corrections are here not needed.

As seen in Fig. 5(a), orbital occupations, with $n_{x y}^{h} \approx 0.2$, are very similar to those found matching values of $\zeta / t_{x z}[43]$. 

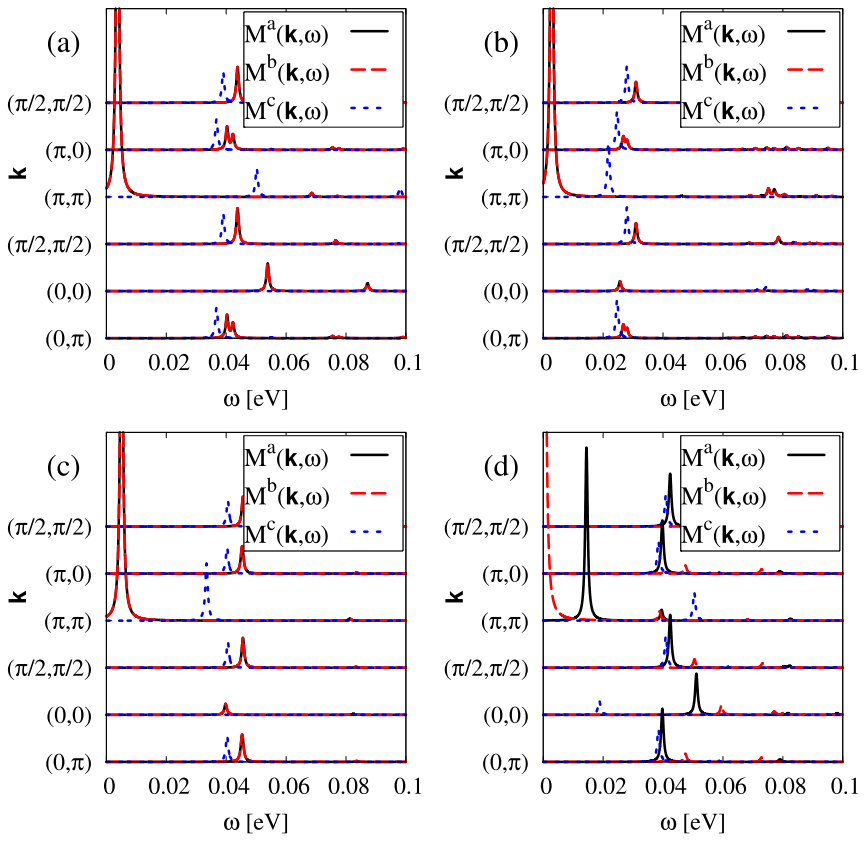

FIG. 6. Dynamic magnetic structure factor (25) for the KugelKhomskii model Eqs. (21) and (20) on an eight-site cluster (a) for parameters obtained to model $\mathrm{Ca}_{2} \mathrm{RuO}_{4}$ (see text), in particular $\zeta=$ $0.13 \mathrm{eV}$ and $\Delta=0.25 \mathrm{eV}$ (b) the same but for reduced $\zeta=0.06 \mathrm{eV}$, (c) as in (a) but increased $\Delta=0.5 \mathrm{eV}$, and (d) as in (a) but with additional magnetic anisotropy $\delta \frac{1}{2}\left(S^{x}-S^{y}\right)^{2}$ favoring moments along the $b$ axis, with $\delta=-0.01 \mathrm{eV}$. Components $M^{a / b / c}(\mathbf{k}, \omega)$ correspond to in-plane transverse $\left(M^{a}\right)$, amplitude $\left(M^{b}\right)$ and out-of-plane transverse $\left(M^{c}\right)$ excitations.

Figure 5(c) also shows that nearly all weight is captured by the onsite singlet $S_{0}$ and doublet $D_{1 / 2}$ and Fig. 5(b) reveals that magnetic ordering transfers weight from $J=0$ to $J=1$, supporting an excitonic-magnetism description. The main impact of the more detailed kinetic energy is that we find the magnetic moment to prefer the $x-y$ direction over the $x$ direction, in agreement with experiment.

\section{Magnetic excitations in $\mathrm{Ca}_{2} \mathrm{RuO}_{4}$}

The original and strongest hint for excitonic magnetism in $\mathrm{Ca}_{2} \mathrm{RuO}_{4}$ is the characteristic neutron-scattering spectrum with its clear maximum at $(0,0)$ indicating pronounced $X Y$ symmetry. While magnetic spectra have been obtained using the VCA [44], the method is not really suitable for twoparticle excitations. We accordingly use the observation that the sum of all the weights $\langle J=0,1,2\rangle$ comes extremely close to one for all parameter sets in Figs. 3(a) and 5(b). This implies that the Hilbert space spanned by these nine states with onsite $S=1$ and $L=1$ is sufficient to describe the ground state.

We thus employ ED to calculate spectra for the KugelKhomskii-like model obtained in second-order perturbation theory in Sec. II B for this limit. We use here the model parameters derived for $\mathrm{Ca}_{2} \mathrm{RuO}_{4}$, but leave off interorbital hopping $t_{o}$ breaking tetragonal symmetry. The spectrum in Fig. 6(a) agrees well with neutron-scattering data for $\mathrm{Ca}_{2} \mathrm{RuO}_{4}[20,26]$ and reproduces the salient feature of a maximum at $(0,0)$

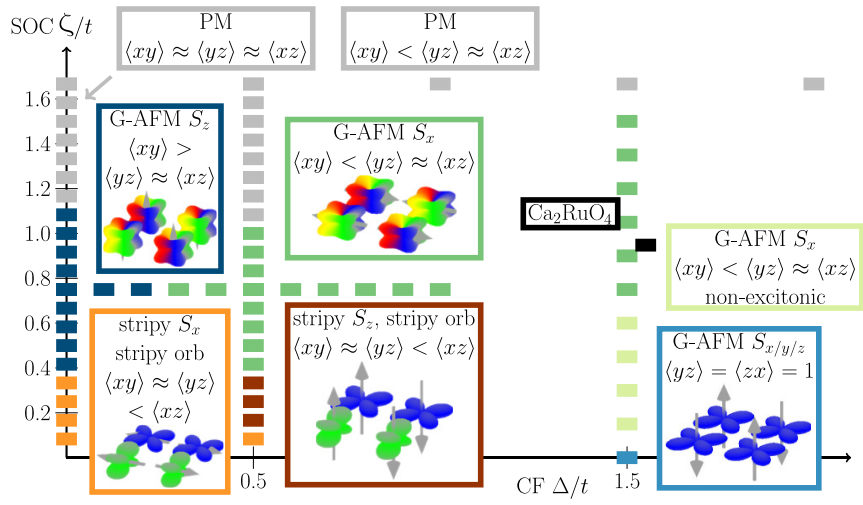

FIG. 7. Approximate phase diagram for the simplified model with $t=t_{x y}^{\mathrm{NN}}=t_{x z}^{\mathrm{NN}}=t_{y z}^{\mathrm{NN}}, t_{x y}^{\mathrm{NNN}}=t^{o}=0, U=12.5 t$, and $J_{H}=$ 2.5t. Expectation values $\langle\beta\rangle$ denote orbital hole densities, with $\beta=y z, x z, x y$. Red and orange denote the stripy spin and orbital order of Fig. 1(b), with red designating a preference for in-plane magnetic ordering and orange for out-of-plane ordering. Dark blue designates the excitonic AFM state with out-of-plane magnetic order and approximately equal hole densities in all orbitals. (For $\Delta=0$, the layered geometry moves slightly more holes into the $x y$ orbital.) Green refers to checkerboard $G$-AFM with in-plane ordered moment and with orbital polarization pushing holes out of the $x y$ orbital. Light green indicates the regime where a description in terms of a spin-one system is more appropriate, and darker green the excitonic regime. The light blue data point indicates the perfectly isotropic Heisenberg spin-one AFM without SOC and with a completely filled $x y$ orbital; gray indicates the PM phase. The approximate place of $\mathrm{Ca}_{2} \mathrm{RuO}_{4}$ in the phase diagram is indicated.

and $\omega \approx 50 \mathrm{meV}$ as well as the dispersion at the zone boundary.

In order to probe the connection of the maximum at $(0,0)$ to SOC, we reduce the latter from $\zeta=0.13 \mathrm{eV}$ to $\zeta=0.06 \mathrm{eV}$, which reduces hole density $n_{x y}^{h}$ from $n_{x y}^{h} \approx 0.25$ to $n_{x y}^{h} \approx 0.11$. At the same time, the maximum at $(0,0)$ disappears, see in Fig. 6(b), where one also notes generally lower excitation energies. Similarly, keeping $\zeta=0.13 \mathrm{eV}$ but increasing $\mathrm{CF}$ from $\Delta=0.25 \mathrm{eV}$ to $\Delta=0.5 \mathrm{eV}$ enhances orbital polarization to $n_{x y}^{h} \approx 0.06$ and at the same time transforms the maximum at $(0,0)$ into a local minimum, see Fig. 6(c).

Finally, we introduce magnetic anisotropy $\delta\left(S^{b}\right)^{2}=$ $\delta \frac{1}{2}\left(S^{x}-S^{y}\right)^{2}$, with $S^{a / b}=\frac{1}{\sqrt{2}}\left(S^{x} \pm S^{y}\right)$ and $\delta=-10 \mathrm{meV}$, to mimic the impact of $t^{o}$, which was found in VCA to prefer moments along the $x-y$ direction. This reproduces the experimentally observed gap of $\omega \approx 15 \mathrm{meV}$ at $(\pi, \pi)$ [45]. The resulting spectrum is given in Fig. $6(\mathrm{~d})$ and while excitation energies are slightly too large by $\approx 10 \%$, it nevertheless agrees well with the data reported in Ref. [20]: In addition to the outof-plane transverse mode $M^{c}$, the broken in-plane symmetry splits the amplitude mode $M^{b}$ off from the in-plane transverse mode $M^{a}$.

\section{Phase diagram}

Going back to the simplified but more generic model without next-nearest-neighbor or interorbital hopping, we give a phase diagram in Fig. 7. For vanishing and small CF and SOC, we find the stripy phase, with a small CF together with 
SOC favoring in-plane magnetism rather than out-of-plane as found for $\Delta=0$. For stronger $\Delta$, magnetic ordering is always checkerboard, but we use shades of green to indicate, where the system can be more appropriately described as a spin-AFM, and where the excitonic description is applicable. Note that there is a regime of SOC $\zeta \approx t-1.5 t$, where applying a $\mathrm{CF} \Delta$ can drive the system through a quantum critical point between the PM and excitonic AFM phases.

\section{SUMMARY AND CONCLUSIONS}

We have used the VCA to investigate excitonic magnetism stabilized by the interplay of superexchange and SOC in strongly correlated $t_{2 g}$ orbitals. Without CF splitting, the main role of SOC is to reduce orbital degeneracy, which in turn changes the magnetic ordering vector from $(0, \pi)$ to $(\pi, \pi)$. A similar transition from a state with complex orbital order to an excitonic antiferromagnet has been found in one dimension [34]. When orbital degeneracy is from the outset reduced by a $\mathrm{CF}$, SOC drives a transition from an orbitally polarized $S=1$ AFM state, where the excitonic picture is not applicable, via excitonic order coexisting with strong orbital polarization, to a paramagnet where SOC suppresses superexchange and thus any magnetic ordering.

As an example, we focus on $\mathrm{Ca}_{2} \mathrm{RuO}_{4}$, which we model using hoppings and $\mathrm{CF}$ strength derived from density-functional theory [27] as well as interaction and SOC parameters inferred from experiment [14]. Magnetic excitations obtained using ED for the corresponding superexchange model are found to closely match neutron-scattering data, in particular showing the telltale maximum at momentum $(0,0)$, and thus validate our microscopic model. We have also shown that this maximum is clearly tied to the small hole density $n_{x y}^{h} \approx 0.2-0.25$ remaining in the $x y$ model. When this is further reduced by smaller SOC or larger $\mathrm{CF}$, the maximum at $(0,0)$ becomes a local minimum.

Strong orbital polarization might suggest a description as an orbitally polarized $S=1$ system, in line with the interpretation of angle-resolved photon-emission-spectroscopy data $[25,46]$. Indeed, the hole densities we find would also support such a picture, as they clearly show that the holes are overwhelmingly found in the $x z$ and $y z$ orbitals with $n_{x z / y z}^{h} \approx 0.9$ vs $n_{x y}^{h} \approx 0.2$. The excitonic character only becomes apparent when looking at two-hole quantities like the total onsite angular momentum or overlap of the onsite density matrix with the states depicted in Fig. 1(a), i.e., lowest eigenstates $S_{0}$ and $D_{1 / 2}$ of the local Hamiltonian (21). While magnetic ordering does not noticeably affect orbital densities, it transfers weight from a singlet onsite ground state $S_{0}$ to doublet $D_{1 / 2}$, as expected for an excitonic magnet. SOC and the remaining small $x y$-hole density thus play a decisive role in the AFM state of $\mathrm{Ca}_{2} \mathrm{RuO}_{4}$, and we conclude $\mathrm{Ca}_{2} \mathrm{RuO}_{4}$ to realize excitonic magnetism despite its pronounced orbital polarization.

\section{ACKNOWLEDGMENTS}

The authors acknowledge support by the state of BadenWürttemberg through bwHPC and via the Center for Integrated Quantum Science and Technology (IQST). M.D. thanks KITP at UCSB for kind hospitality, this research was thus supported in part by the National Science Foundation under Grant No. NSF PHY-1748958.
[1] D. Pesin and L. Balents, Mott physics and band topology in materials with strong spin-orbit interaction, Nat. Phys. 6, 376 (2010).

[2] A. Kitaev, Anyons in an exactly solved model and beyond, Ann. Phys. 321, 2 (2006).

[3] G. Jackeli and G. Khaliullin, Mott Insulators in the Strong SpinOrbit Coupling Limit: From Heisenberg to a Quantum Compass and Kitaev Models, Phys. Rev. Lett. 102, 017205 (2009).

[4] J. Chaloupka, G. Jackeli, and G. Khaliullin, Kitaev-Heisenberg Model on a Honeycomb Lattice: Possible Exotic Phases in Iridium Oxides $\mathrm{A}_{2} \mathrm{IrO}_{3}$, Phys. Rev. Lett. 105, 027204 (2010).

[5] S. M. Winter, A. A. Tsirlin, M. Daghofer, J. van den Brink, Y. Singh, P. Gegenwart, and R. Valenti, Models and materials for generalized Kitaev magnetism, J. Phys. Condens. Matter 29, 493002 (2017).

[6] H. Takagi, T. Takayama, G. Jackeli, G. Khaliullin, and S. E. Nagler, Kitaev quantum spin liquid-Concept and materialization, Nat. Rev. Phys. 1, 264 (2019).

[7] G. Khaliullin, Orbital order and fluctuations in Mott insulators, Progr. Theor. Phys. Suppl. 160, 155 (2005).

[8] J. Bertinshaw, Y. Kim, G. Khaliullin, and B. Kim, Square lattice iridates, Annu. Rev. Condens. Matter Phys. 10, 315 (2019).
[9] Y. K. Kim, O. Krupin, J. D. Denlinger, A. Bostwick, E. Rotenberg, Q. Zhao, J. F. Mitchell, J. W. Allen, and B. J. Kim, Fermi arcs in a doped pseudospin-1/2 Heisenberg antiferromagnet, Science 345, 187 (2014).

[10] Y. K. Kim, N. H. Sung, J. D. Denlinger, and B. J. Kim, Observation of a $d$-wave gap in electron-doped $\mathrm{Sr}_{2} \mathrm{IrO}_{4}$, Nat. Phys. 12, 37 (2015).

[11] D. Afanasiev, A. Gatilova, D. J. Groenendijk, B. A. Ivanov, M. Gibert, S. Gariglio, J. Mentink, J. Li, N. Dasari, M. Eckstein, T. Rasing, A. D. Caviglia, and A. V. Kimel, Ultrafast Spin Dynamics in Photodoped Spin-Orbit Mott Insulator $\mathrm{Sr}_{2} \mathrm{IrO}_{4}$, Phys. Rev. X 9, 021020 (2019).

[12] K. Pajskr, P. Novák, V. Pokorný, J. Kolorenč, R. Arita, and J. Kuneš, On the possibility of excitonic magnetism in Ir double perovskites, Phys. Rev. B 93, 035129 (2016).

[13] S. Fuchs, T. Dey, G. Aslan-Cansever, A. Maljuk, S. Wurmehl, B. Büchner, and V. Kataev, Unraveling the Nature of Magnetism of the $5 d^{4}$ Double Perovskite $\mathrm{Ba}_{2} \mathrm{YIrO}_{6}$, Phys. Rev. Lett. 120, 237204 (2018).

[14] H. Gretarsson, H. Suzuki, H. Kim, K. Ueda, M. Krautloher, B. J. Kim, H. Yavaş, G. Khaliullin, and B. Keimer, Observation of spin-orbit excitations and Hund's multiplets in $\mathrm{Ca}_{2} \mathrm{RuO}_{4}$, Phys. Rev. B 100, 045123 (2019). 
[15] G. Khaliullin, Excitonic Magnetism in Van Vleck-Type $d^{4}$ Mott Insulators, Phys. Rev. Lett. 111, 197201 (2013).

[16] O. N. Meetei, W. S. Cole, M. Randeria, and N. Trivedi, Novel magnetic state in $d^{4}$ Mott insulators, Phys. Rev. B 91, 054412 (2015).

[17] P. S. Anisimov, F. Aust, G. Khaliullin, and M. Daghofer, Nontrivial Triplon Topology and Triplon Liquid in KitaevHeisenberg-Type Excitonic Magnets, Phys. Rev. Lett. 122, 177201 (2019).

[18] J. Chaloupka and G. Khaliullin, Highly frustrated magnetism in relativistic $d^{4}$ Mott insulators: Bosonic analog of the Kitaev honeycomb model, Phys. Rev. B 100, 224413 (2019).

[19] J. Chaloupka and G. Khaliullin, Doping-Induced Ferromagnetism and Possible Triplet Pairing in $d^{4}$ Mott Insulators, Phys. Rev. Lett. 116, 017203 (2016).

[20] A. Jain, M. Krautloher, J. Porras, G. Ryu, D. Chen, D. Abernathy, J. Park, A. Ivanov, J. Chaloupka, G. Khaliullin, B. Keimer, and B. Kim, Nat. Phys. 13, 633 (2017).

[21] S.-M. Souliou, J. Chaloupka, G. Khaliullin, G. Ryu, A. Jain, B. J. Kim, M. Le Tacon, and B. Keimer, Raman Scattering from Higgs Mode Oscillations in the Two-Dimensional Antiferromagnet $\mathrm{Ca}_{2} \mathrm{RuO}_{4}$, Phys. Rev. Lett. 119, 067201 (2017).

[22] I. Zegkinoglou, J. Strempfer, C. S. Nelson, J. P. Hill, J. Chakhalian, C. Bernhard, J. C. Lang, G. Srajer, H. Fukazawa, S. Nakatsuji, Y. Maeno, and B. Keimer, Orbital Ordering Transition in $\mathrm{Ca}_{2} \mathrm{RuO}_{4}$ Observed with Resonant X-Ray Diffraction, Phys. Rev. Lett. 95, 136401 (2005).

[23] T. Mizokawa, L. H. Tjeng, G. A. Sawatzky, G. Ghiringhelli, O. Tjernberg, N. B. Brookes, H. Fukazawa, S. Nakatsuji, and Y. Maeno, Spin-Orbit Coupling in the Mott Insulator $\mathrm{Ca}_{2} \mathrm{RuO}_{4}$, Phys. Rev. Lett. 87, 077202 (2001).

[24] G. Zhang and E. Pavarini, Mott transition, spin-orbit effects, and magnetism in $\mathrm{Ca}_{2} \mathrm{RuO}_{4}$, Phys. Rev. B 95, 075145 (2017).

[25] D. Sutter, C. G. Fatuzzo, S. Moser, M. Kim, R. Fittipaldi, A. Vecchione, V. Granata, Y. Sassa, F. Cossalter, G. Gatti, M. Grioni, H. M. Rønnow, N. C. Plumb, C. E. Matt, M. Shi, M. Hoesch, T. K. Kim, T.-R. Chang, H.-T. Jeng, C. Jozwiak, A. Bostwick, E. Rotenberg, A. Georges, T. Neupert, and J. Chang, Hallmarks of Hunds coupling in the Mott insulator $\mathrm{Ca}_{2} \mathrm{RuO}_{4}$, Nat. Commun. 8, 15176 (2017).

[26] S. Kunkemöller, D. Khomskii, P. Steffens, A. Piovano, A. A. Nugroho, and M. Braden, Highly Anisotropic Magnon Dispersion in $\mathrm{Ca}_{2} \mathrm{RuO}_{4}$ : Evidence for Strong Spin Orbit Coupling, Phys. Rev. Lett. 115, 247201 (2015).

[27] J. Bertinshaw, N. Gurung, P. Jorba, H. Liu, M. Schmid, D. T. Mantadakis, M. Daghofer, M. Krautloher, A. Jain, G. H. Ryu, O. Fabelo, P. Hansmann, G. Khaliullin, C. Pfleiderer, B. Keimer, and B. J. Kim, Unique Crystal Structure of $\mathrm{Ca}_{2} \mathrm{RuO}_{4}$ in the Current Stabilized Semimetallic State, Phys. Rev. Lett. 123, 137204 (2019).

[28] M. Cuoco, F. Forte, and C. Noce, Probing spin-orbital-lattice correlations in $4 d^{4}$ systems, Phys. Rev. B 73, 094428 (2006).
[29] R. Triebl, G. J. Kraberger, J. Mravlje, and M. Aichhorn, Spinorbit coupling and correlations in three-orbital systems, Phys. Rev. B 98, 205128 (2018).

[30] A. M. Oleś, Antiferromagnetism and correlation of electrons in transition metals, Phys. Rev. B 28, 327 (1983).

[31] M. Potthoff, M. Aichhorn, and C. Dahnken, Variational Cluster Approach to Correlated Electron Systems in Low Dimensions, Phys. Rev. Lett. 91, 206402 (2003).

[32] H. Watanabe, T. Shirakawa, and S. Yunoki, Microscopic Study of a Spin-Orbit-Induced Mott Insulator in Ir Oxides, Phys. Rev. Lett. 105, 216410 (2010).

[33] N. Kaushal, R. Soni, A. Nocera, G. Alvarez, and E. Dagotto, BCS-BEC crossover in a $\left(t_{2 g}\right)^{4}$ excitonic magnet, Phys. Rev. B 101, 245147 (2020).

[34] N. Kaushal, J. Herbrych, A. Nocera, G. Alvarez, A. Moreo, F. A. Reboredo, and E. Dagotto, Density matrix renormalization group study of a three-orbital Hubbard model with spin-orbit coupling in one dimension, Phys. Rev. B 96, 155111 (2017).

[35] M. Balzer and M. Potthoff, Variational cluster approach to ferromagnetism in infinite dimensions and in one-dimensional chains, Phys. Rev. B 82, 174441 (2010).

[36] M. Potthoff, Self-energy-functional approach: Analytical results and the Mott-Hubbard transition, Eur. Phys. J. B 36, 335 (2003).

[37] M. Aichhorn and E. Arrigoni, Weak phase separation and the pseudogap in the electron-doped cuprates, Eurphys. Lett. 72, 117 (2005).

[38] T. Hotta and E. Dagotto, Prediction of Orbital Ordering in Single-Layered Ruthenates, Phys. Rev. Lett. 88, 017201 (2001).

[39] M. Cuoco, F. Forte, and C. Noce, Interplay of Coulomb interactions and $c$-axis octahedra distortions in single-layer ruthenates, Phys. Rev. B 74, 195124 (2006).

[40] T. Feldmaier, Excitonic Antiferromagnetism in Two-dimensional $t_{2 g}^{4}$ Systems, Ph.D. thesis, University of Stuttgart (2019).

[41] G. Khaliullin and S. Okamoto, Quantum Behavior of Orbitals in Ferromagnetic Titanates: Novel Orderings and Excitations, Phys. Rev. Lett. 89, 167201 (2002).

[42] T. Sato, T. Shirakawa, and S. Yunoki, Spin-orbital entangled excitonic insulator with quadrupole order, Phys. Rev. B 99, 075117 (2019).

[43] $t_{x z}^{\mathrm{NN}}=t_{y z}^{\mathrm{NN}}$ are the two presumably most relevant hopping channels as they connect approximately half-filled orbitals.

[44] S. Brehm, E. Arrigoni, M. Aichhorn, and W. Hanke, Theory of two-particle excitations and the magnetic susceptibility in high-tccuprate superconductors, Eurphys. Lett. 89, 27005 (2010).

[45] Note that a finite cluster cannot show spontaneous symmetry breaking.

[46] A. Kłosiński, D. V. Efremov, J. van den Brink, and K. Wohlfeld, Photoemission spectrum of $\mathrm{Ca}_{2} \mathrm{RuO}_{4}$ : Spin polaron physics in an $S=1$ antiferromagnet with anisotropies, Phys. Rev. B 101, 035115 (2020). 